\title{
Ward Capacity Strain: A Novel Predictor of 30-Day Hospital Readmissions
}

\author{
Rachel Kohn, MD, MSCE ${ }^{1,2,3,4,5}$, Michael O. Harhay, PhD ${ }^{3,4,6}$, Brian Bayes, $M S^{2,4}$, \\ Mark E. Mikkelsen, MD, MSCE ${ }^{1,4,5}$, Sarah J. Ratcliffe, $P h D^{7}$, Scott D. Halpern, MD, PhD ${ }^{1,2,3,4,5}$, and \\ Meeta Prasad Kerlin, MD, MSCE $E^{1,2,3,4,5}$
}

\begin{abstract}
'Department of Medicine, Perelman School of Medicine at the University of Pennsylvania, Philadelphia, PA, USA; ${ }^{2}$ Center for Clinical Epidemiology and Biostatistics, Perelman School of Medicine at the University of Pennsylvania, Philadelphia, PA, USA; ${ }^{3}$ Leonard Davis Institute of Health

Economics, Perelman School of Medicine at the University of Pennsylvania, Philadelphia, PA, USA; ${ }^{4}$ Palliative and Advanced Illness Research (PAIR) Center at the University of Pennsylvania, Philadelphia, PA, USA; ${ }^{5}$ Hospital of the University of Pennsylvania, Philadelphia, PA, USA; ${ }^{6}$ Department of Biostatistics, Epidemiology and Informatics, Perelman School of Medicine at the University of Pennsylvania, Philadelphia, PA, USA; ${ }^{7}$ Department of Public Health Sciences and Division of Biostatistics at the University of Virginia, Charlottesville, VA, USA.
\end{abstract}

KEY WORDS: capacity; workload; crowding; general wards; 30-day hospital readmissions.

J Gen Intern Med 33(11):1851-3

DOI: $10.1007 / \mathrm{s} 11606-018-4564-\mathrm{x}$

(c) Society of General Internal Medicine 2018

\section{INTRODUCTION}

The Centers for Medicare and Medicaid Services' Hospital Readmissions Reduction Program penalizes hospitals for excess 30-day hospital readmissions. ${ }^{1}$ Readmission reduction efforts have focused on patient-level factors. Whether organizational factors contribute to readmission risk is largely unknown. Intensive care unit (ICU) survivors are particularly at risk for hospital readmissions. ${ }^{2,3}$ ICU capacity strain, when demand for ICU resources exceeds availability, is associated with short-term ICU patient outcomes. ${ }^{4,5}$ However, most ICU survivors are transferred to general wards prior to hospital discharge and are therefore subjected to ward-level exposures that could impact readmission risk.

Our objective was to determine if ward capacity $\operatorname{strain}^{6}$ on the last day of hospitalization is associated with increased 30day hospital readmission rates, as pressures on staff and resources may impede optimal discharge planning for complex patients.

\section{METHODS}

We performed a retrospective cohort study in three organizationally independent University of Pennsylvania Health System hospitals with diverse patient populations. We included data from electronic medical records for medical and surgical ICU survivors discharged to general wards in 2014 and 2015. We excluded patients who died prior to discharge, transitioned to hospice, left against medical advice, and were transferred to other acute care hospitals.

We defined ward capacity strain by patient volume, staff workload, and ward acuity measures on patients' last calendar

Published online July 18, 2018 day of hospitalization. Patient volume measures included numbers of new admissions and hospital discharges, and census (number of patients occupying beds). Staff workload measures included numbers of off-ward transports, total medications administered across patients, orders requiring respiratory therapy involvement, and blood products transfused. Overall acuity measures included numbers of patients on telemetry monitoring, with quick Sequential Organ Failure Assessment (qSOFA) scores $\geq 2$, and transferred to an ICU. ${ }^{6}$

The outcome was 30-day all-cause hospital readmissions. We performed logistic regression using pre-specified patient characteristics (age, gender, race, insurance type, Elixhauser comorbidities, and pre-ward hospital length of stay) to predict 30-day hospital readmissions overall and stratified by individual wards. We then added ward-level strain variables and repeated logistic regression to predict 30-day hospital readmissions overall and stratified by ward. We used cstatistics to assess model discrimination and HosmerLemeshow (H-L) chi-squared goodness-of-fit tests to assess calibration. We used DeLong tests for each set of paired models to compare discrimination. We then performed penalized logistic regression using least absolute shrinkage and selection operator, including patient- and ward-level variables to identify variables most predictive of 30-day hospital readmissions.

We performed all analyses using Stata version 14.1 (StataCorp LP, College Station, TX). The University of Pennsylvania Institutional Review Board approved this protocol.

\section{RESULTS}

The study population included 18,710 visits among 12,429 ICU survivors in 33 wards. Median age was 63 years (IQR $51-73), 56 \%$ were male, and $61 \%$ were white. Two thousand six hundred ten (14\%) visits were readmitted within 30 days of hospital discharge (11-226 visits per ward). Of the 33 wards, there were 32 median operational beds (IQR 30-42) per ward. 


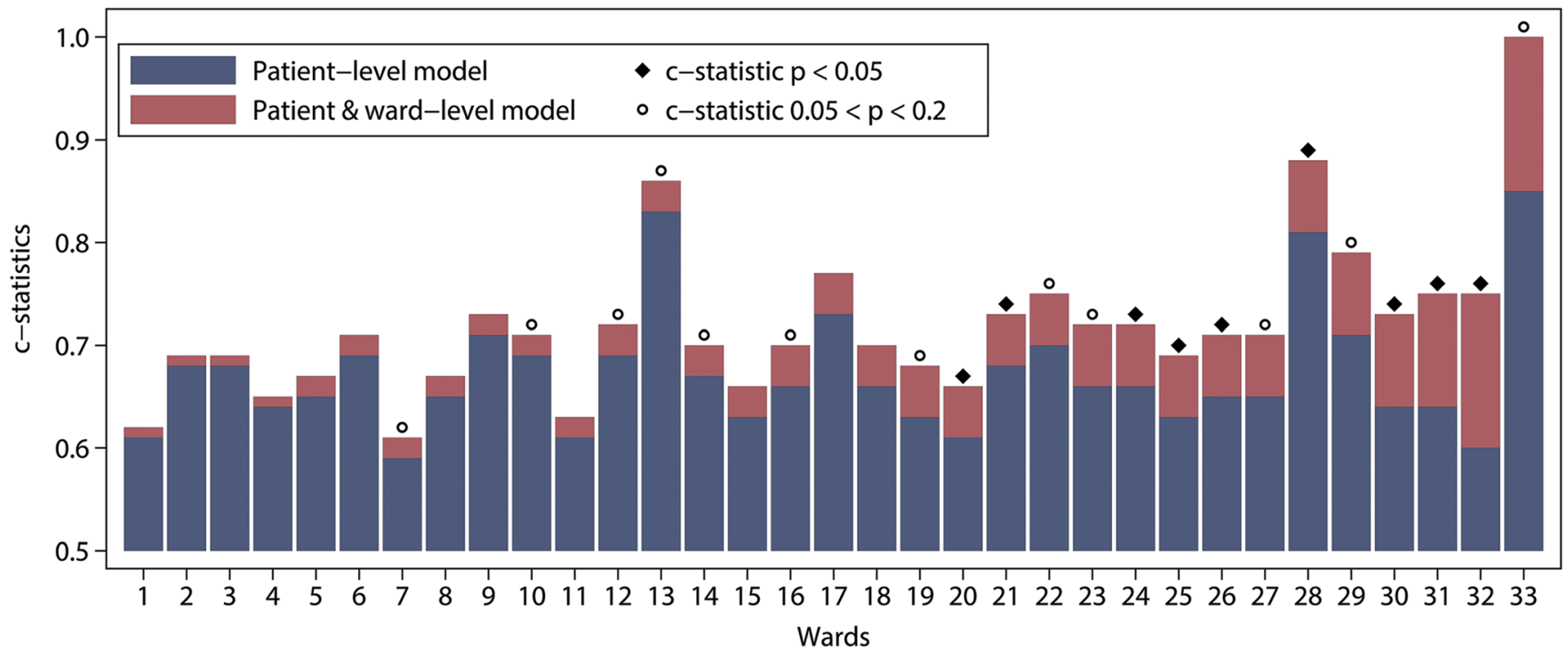

Fig. 1 Heterogeneous increases in c-statistics across wards when ward-level capacity strain variables are added to patient-level variables.**The c-statistic is a measure of predictive accuracy that ranges from 0.5 to 1.0 . Generally, a statistical model is considered to be reasonable when the c-statistic is higher than 0.7 and strong when the c-statistic exceeds 0.8 . An increase in the c-statistic for a given ward indicates that the inclusion of measures of ward capacity strain improved the prediction of the statistical model over the model without these variables. The result of the statistical tests to quantify these increases is indicated in the figure legend.

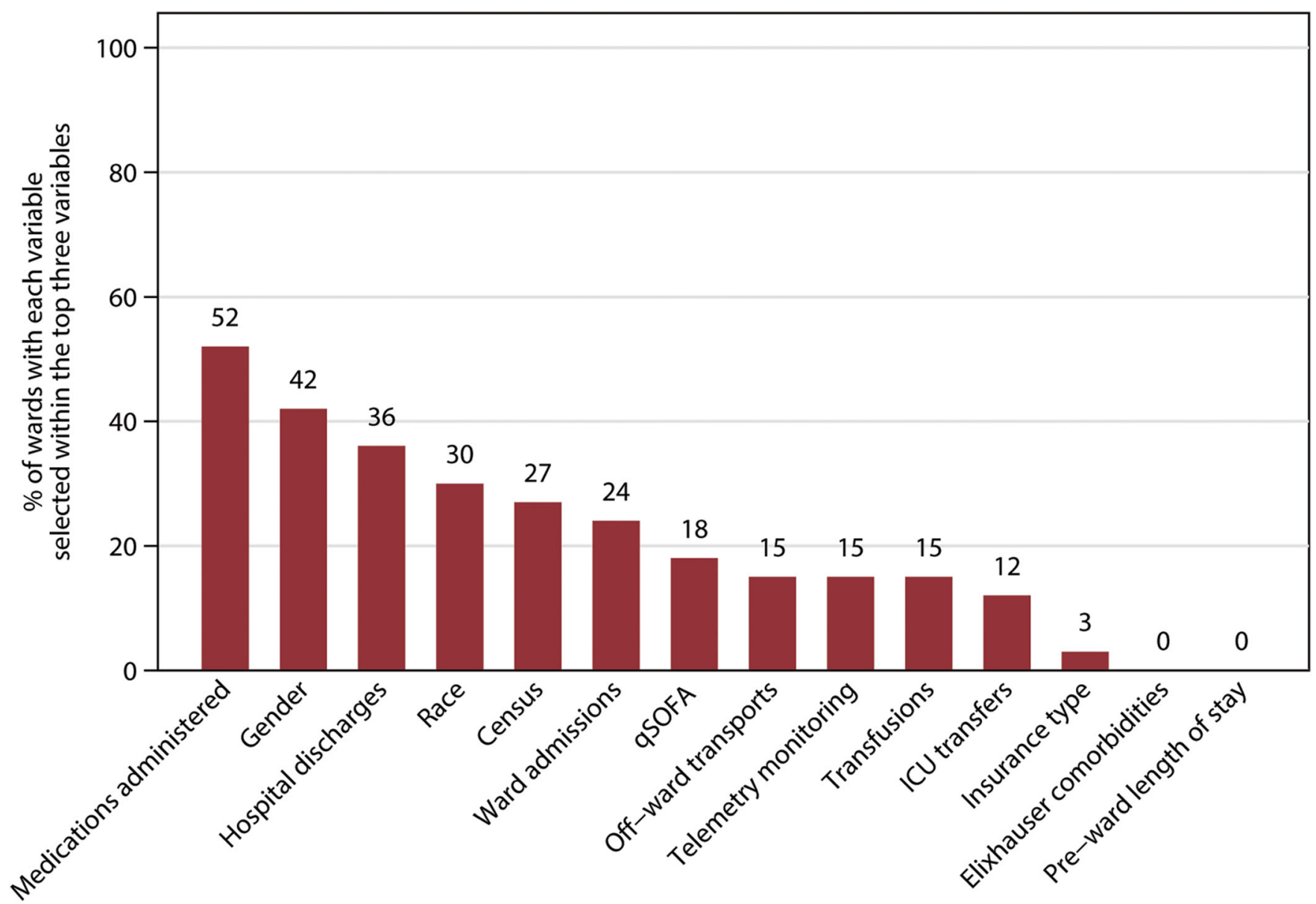

Fig. 2 Medications administered, hospital discharges, and census are three of the five strongest predictors of 30-day hospital readmissions across individual wards.* *In penalized logistic regression, variables enter the model according to predictive value. Variables that are added in first, second, and third, for example, can be interpreted as the three strongest predictors of the outcome, which are presented here. qSOFA, quick Sequential Organ Failure Assessment; ICU, intensive care unit. 
In the population overall, models with patient characteristics alone and with the addition of ward-level variables had similar predictive ability (c-statistic 0.61 [95\% CI 0.59-0.61] versus 0.62 [95\% CI 0.60-0.63], respectively; $p=0.03$ ). However, among individual wards, the addition of ward-level variables improved predictive ability (c-statistics increased by $0.01-0.15$ ), with 9 of 33 wards having significant increases according to DeLong tests (Fig. 1). Thirty-one of 33 wards had non-significant $\mathrm{H}-\mathrm{L}$ tests, indicating good model calibration. Medications administered, hospital discharges, and census were three of the five strongest predictors of 30-day hospital readmissions (Fig. 2).

\section{DISCUSSION}

In an integrated health system, although unchanged in the overall population, ward capacity strain metrics improved prediction of 30-day hospital readmission risk in nearly one out of three hospital wards, likely due to the heterogeneity of the overall population. This study has important limitations. First, adjustment for patient case-mix may have been imperfect. Second, small numbers of ICU survivors per ward could lead to model overfitting. Last, readmissions to other hospital systems and emergency department (ED) visits were not captured, which would bias our findings towards the null. If we included readmissions to other hospital systems and EDs, our findings would only be more robust. Future studies are needed to externally validate our findings and to assess the impact of ward capacity strain on hospital readmissions among other patient populations and in other settings.

Corresponding Author: Rachel Kohn, MD, MSCE; Hospital of the University of Pennsylvania, Philadelphia, PA 19104, USA (e-mail: rachel.kohn2@uphs.upenn.edu).
Authors' Contributions All of the authors made substantial contributions to the conception or design of the study, or the acquisition, analysis, or interpretation of data, and drafting or revising the manuscript.

Funding Dr. Kohn was supported by NIH/NHLBI T32 HL007891 and F32 HL139107-01. Dr. Harhay was supported by NIH/NHLBI K99 HL141678. Dr. Kerlin was supported by NIH/NHLBI KO8 HL116771.

\section{Compliance with Ethical Standards:}

Conflict of Interest: The authors declare that they do not have conflicts of interest.

\section{REFERENCES}

1. Centers for Medicare \& Medicaid Services Readmissions Reduction Program (HRRP). Last modified April 27, 2018: https://www.cms.gov/ medicare/medicare-fee-for-service-payment/acuteinpatientpps / readmissions-reduction-program.html. Accessed June 15, 2018.

2. Hua M, Gong MN, Brady J, Wunsch H. Early and late unplanned rehospitalizations for survivors of critical illness. Crit Care Med. 2015;43(2):430-438.

3. Garland A, Olafson $\mathbf{K}$, Ramsey CD, Yogendran $\mathbf{M}$, Fransoo R. A population-based observational study of intensive care unit-related outcomes. With emphasis on post-hospital outcomes. Ann Am Thorac Soc. 2015; 12(2):202-208.

4. Gabler NB, Ratcliffe SJ, Wagner $\mathbf{J}$, et al. Mortality among patients admitted to strained intensive care units. Am J Respir Crit Care Med. 2013; 188(7):800-806.

5. Wagner J, Gabler NB, Ratcliffe SJ, Brown SE, Strom BL, Halpern SD. Outcomes among patients discharged from busy intensive care units. Ann Intern Med. 2013;159(7):447-455.

6. Kohn R, Bayes B, Ratcliffe SJ, Halpern SD, Kerlin MP. Ward Capacity Strain: Defining a New Construct Based on ED Boarding Time and ICU Transfers. Presented at: American Thoracic Society International Conference 2017; Washington, DC. 